\title{
Alterações oxidativas (cor e lipídios) em presunto de peru tratado por Alta Pressão Hidrostática (APH)
}

\author{
The oxidative alterations (color and lipids) on turkey ham treated by High Hydrostatic Pressure (HHP)
}

Simone Pereira MATHIAS ${ }^{6 *}$, Amauri ROSENTHAL ${ }^{1}$, Arlene GASPAR ${ }^{7}$, Rosires DELIZA ${ }^{2}$, Adriana Paula SLONGO ${ }^{5}$, Juarez VICENTE 6 , Lourdes Maria MASSON ${ }^{3}$, Celso BARBOSA ${ }^{4}$

\begin{abstract}
Resumo
A Alta Pressão Hidrostática (APH) consiste em uma tecnologia inovadora de processamento de alimentos com utilização de pressões elevadas (100 a $900 \mathrm{MPa}$ ) que possui vantagens em relação às tecnologias térmicas convencionais, pois mantém as características sensoriais do alimento próximas do original e garante segurança do ponto de vista microbiológico, por prolongado período de vida útil. No presente estudo, foi avaliada a aplicação de alta pressão a presunto de peru nos processos oxidativos, em particular na oxidação de lipídios e os efeitos na cor do produto, quando tratados a pressões de 200 a $400 \mathrm{MPa}$, por 5, 10 e 15 minutos em temperatura ambiente. Dos resultados obtidos, pode-se observar que não foram verificadas diferenças significativas $(\mathrm{p}>0,05)$ entre amostras controle e pressurizada para o índice de TBA e índice de peróxido. Foi observado também alteração da cor decorrente de reações oxidativas aos 45 e 65 dias de armazenamento em amostras tratadas com pressões de $400 \mathrm{MPa}$ a 5 e 15 minutos.
\end{abstract}

Palavras-chave: alta pressão hidrostática; presunto de peru; oxidação de lipídios e cor.

\begin{abstract}
The High Hydrostatic Pressure (HHP) is an innovative food technology which uses high pressure (100 a $900 \mathrm{MPa})$ and has the advantage over conventional thermal technologies of keeping the sensory characteristics of food similar to the original characteristics ensuring the food safety, from the microbiological point of view, for an increased shelf life. This study aimed at investigating the effects of high pressure on the oxidative process, particularly on the lipid oxidation, and their effects on the color of pressurized turkey ham when treated from 200 to $400 \mathrm{MPa}$ for 5, 10, and 15 minutes at room temperature. From the results, one can observe that no significant difference ( $\mathrm{p}>0.05$ ) was found between the control and pressurized samples for the TBA indexes, but a difference was observed in the peroxide index on the $30^{\text {th }}$ day among the samples. It was also observed change of color due to oxidative reactions after 45 and 65 days of storage in the samples treated under pressures of $400 \mathrm{MPa}$ for 5 and 15 minutes.

Keywords: high hydrostatic pressure; ham turkey; oxidation of lipids and color.
\end{abstract}

\section{Introdução}

A tecnologia de Alta Pressão Hidrostática (APH) temse destacado como inovadora, englobando os requisitos de uma tecnologia que preserva a qualidade do produto, sem acarretar danos ao meio ambiente. Trata-se de um tratamento não térmico e que utiliza pressões elevadas, na faixa de 100 a $900 \mathrm{MPa}$, com opções concomitantes de variação de tempo e temperatura, o que garante uma flexibilidade de trabalho de acordo com o alimento utilizado. Possui grande vantagem de aumentar a vida útil, garantir um alimento seguro do ponto de vista microbiológico, além de manter as características nutricionais e sensoriais praticamente inalteradas. Os custos envolvidos na aquisição dos equipamentos e do processamento limitam o uso dessa tecnologia. Avanços têm sido realizados no desenho e construção desses equipamentos para tornar tais custos de processo mais competitivos em relação à esterilização e ao congelamento. Alguns produtos tratados pela alta pressão hidrostática já estão sendo comercializados na Alemanha, Espanha, Estados Unidos e Japão, e a tendência é de que outros países venham a adquirir essa tecnologia que traz grandes vantagens para o processamento e conservação dos alimentos.

Os produtos cárneos, devido à sua riqueza na composição em relação à umidade, proteínas, gorduras e outros nutrientes, são produtos bastante susceptíveis às alterações de ordem física, química e microbiológica (OLIVO, 2006). Um dos maiores desafios para a indústria de carnes é oferecer produtos macios, suculentos e com cor e sabor agradáveis, permanecendo estáveis essas características de frescor durante toda a sua vida de prateleira, com a maior segurança e o menor custo possível.

\footnotetext{
Recebido para publicação em 4/9/2008

Aceito para publicação em 16/10/2009 (003268)

1 Departamento CTAA, EMBRAPA, CEP 23020-470, Rio de Janeiro - RJ, Brasil

2 Laboratório de Análise Sensorial e Instrumental, EMBRAPA, Agroindústria de Alimentos, CEP 23020-470, Rio de Janeiro - RJ, Brasil

${ }^{3}$ Departamento de Engenharia Química, Universidade Federal do Rio de Janeiro - UFRJ, Escola de Química, Rio de Janeiro - RJ, Brasil

${ }^{4}$ Departamento de Matemática e Estatística, Universidade Federal Rural do Rio de Janeiro - UFRRJ, CEP 23890-000, Seropédica - RJ, Brasil

${ }^{5}$ Departamento de Engenharia de Alimentos, Universidade Federal de Santa Catarina - UFSC, Florianópolis - SC, Brasil

${ }^{6}$ Departamento de Tecnologia de Alimentos, Universidade Federal Rural do Rio de Janeiro - UFRRJ, Seropédica - RJ, Brasil, E-mail: spmathias@terra.com.br

Departamento de Tecnologia de Alimentos, Universidade Federal Rural do Rio de Janeiro - UFRRJ, CEP 23890-000, Seropédica - RJ, Brasil

${ }^{*}$ A quem a correspondência deve ser enviada
} 
A oxidação lipídica é uma das mais importantes causas da deterioração de alimentos, podendo conduzir à perda de cor, sabor, modificação da textura e formação de compostos tóxicos. A oxidação de lipídios e a alteração da cor causada por reações oxidativas são difíceis de serem controladas, principalmente devido à complexidade e variabilidade das reações envolvidas. Tais reações são de ordem físico-química, podendo ser potencializadas por ação microbiológica (SHIMOKOMAKI; OLIVO, 2006).

$\mathrm{O}$ processo a $\mathrm{APH}$, embora preserve de modo geral a qualidade e garanta a segurança do produto, pode conduzir a mudanças na estrutura da carne e causar efeitos adversos em alguns parâmetros desejáveis, como a cor (RUBIO et al., 2007). O objetivo do presente estudo foi avaliar o efeito da aplicação da tecnologia de alta pressão hidrostática sobre presunto de peru, em particular no tocante à estabilidade de cor do produto e ocorrência de oxidação de lipídios durante a vida de prateleira.

\section{Material e métodos}

\subsection{Matéria-prima}

Coxas de peru congeladas, embaladas em sacos plásticos (embalagem primária), contendo aproximadamente $1,2 \mathrm{~kg}$ e acondicionadas em caixas de papelão (embalagem secundária) com $15 \mathrm{~kg}$ cada, foram adquiridas de empresa sediada no Sul do Brasil com distribuição no Rio de Janeiro e usadas como matéria-prima para o processamento. A empresa trabalha com cortes especiais de peru congelado e realizou a entrega da mercadoria em caminhão com temperatura controlada, seguindo todos os requisitos básicos de higiene e conservação. Realizada a entrega, a mercadoria foi armazenada à temperatura de $-18{ }^{\circ} \mathrm{C}$, em câmara frigorífica, na Planta II da Embrapa Agroindústria de Alimentos.

\subsection{Processamento do presunto de peru}

Para a fabricação do presunto de peru, foi realizado o toalete na coxa de peru congelada com facas do tipo desossa, procedendo-se à retirada dos tendões, nervos, pele e ossos e cortando a carne em pedaços menores. Para a formulação (Tabela 1), os componentes da salmoura foram pesados em balança analítica e diluídos em água gelada em recipiente plástico, mantidos sob agitação constante, para completa dissolução, até que fossem adicionados à carne.

Tabela 1. Formulação do presunto de peru.

\begin{tabular}{lc}
\hline \multicolumn{1}{c}{ Formulação } & Quantidade \\
\hline Coxa de peru em pedaços & $2,5 \mathrm{~kg}$ \\
Supergal $^{\circledast} 202 / 11^{*}$ & $78,025 \mathrm{~g}$ \\
${\text { Condimento Misto para Presunto Califórnia } 616 / 1^{\circledR}}^{\star}$ & $26,250 \mathrm{~g}$ \\
Realçador de Sabor $404^{\circledast}$ & $10,625 \mathrm{~g}$ \\
Açúcar & $10,675 \mathrm{~g}$ \\
Sal & $37,500 \mathrm{~g}$ \\
Água gelada & $950 \mathrm{~mL}$ \\
\hline
\end{tabular}

*Produto da Empresa Duas Rodas formulado com sal, açúcar, conservador nitrito de sódio, estabilizante polifosfatos, antioxidante eritorbato de sódio.
Em seguida, misturou-se a carne e a salmoura, levando-as ao cutter (marca Geiger e modelo UM12), intercalando-se a operação com 2 a 3 intervalos de alguns segundos, com o intuito de reduzir a carne a pedaços menores até a obtenção de uma massa mais homogênea. A massa foi posteriormente transferida para um recipiente plástico com tampa e levada à geladeira $5^{\circ} \mathrm{C}$, permanecendo por 24 horas. Após este período, a massa obtida, contendo em média $2,5 \mathrm{~kg}$, foi colocada em embalagem plástica resistente à alta temperatura (cook-in), fechada em seladora a vácuo (marca Engevac e modelo 30 gás) e posteriormente acondicionada em formas de aço inoxidável para o cozimento. O cozimento foi realizado em autoclave com calor úmido até que a temperatura interna do produto atingisse no mínimo $68^{\circ} \mathrm{C}$, cuja verificação foi feita com controlador interno de temperatura (modelo ELLAB) em umas das peças controle. Após o cozimento, o produto foi resfriado em banho de gelo por 40 minutos e, posteriormente, armazenado em geladeira a $5^{\circ} \mathrm{C}$ durante 24 horas. Em seguida, o presunto de peru foi fatiado e posteriormente embalado a vácuo. Para o fatiamento, foi utilizado um fatiador de frios (marca SKYMSEN, modelo CFI-300) previamente higienizado. As peças de presunto a serem utilizadas também tiveram sua película plástica higienizada e depois removida, para proceder ao corte em fatias de 0,5 mm de espessura. Feito este procedimento, as fatias foram acondicionadas em sacos plásticos estéreis, medindo em média $10 \times 4 \mathrm{~cm}$, selados a vácuo e enviadas para pressurização (Figura 1).

\subsection{Tratamento a alta pressão hidrostática}

As amostras fatiadas e embaladas a vácuo foram submetidas à alta pressão no equipamento estático da Embrapa Agroindústria de Alimentos (marca Stansted Fluid Power, modelo S-FL-8509-W), o qual possui capacidade para operar em faixa de pressão de 100 a $600 \mathrm{MPa}$ em temperaturas variando de 0 a $80{ }^{\circ} \mathrm{C}$ e intervalos de tempos variados. $\mathrm{O}$ equipamento foi controlado através de um painel digital para o ajuste da pressão, tempo e temperatura desejada, de acordo com o planejamento experimental. Posteriormente, as amostras do presunto de peru foram colocadas dentro do cilindro interno, de aço inoxidável, de aproximadamente $7,0 \mathrm{~cm}$ de diâmetro e $20,0 \mathrm{~cm}$ de comprimento, e que possui vários orifícios por onde circula o líquido pressurizador, neste caso álcool 70\%.

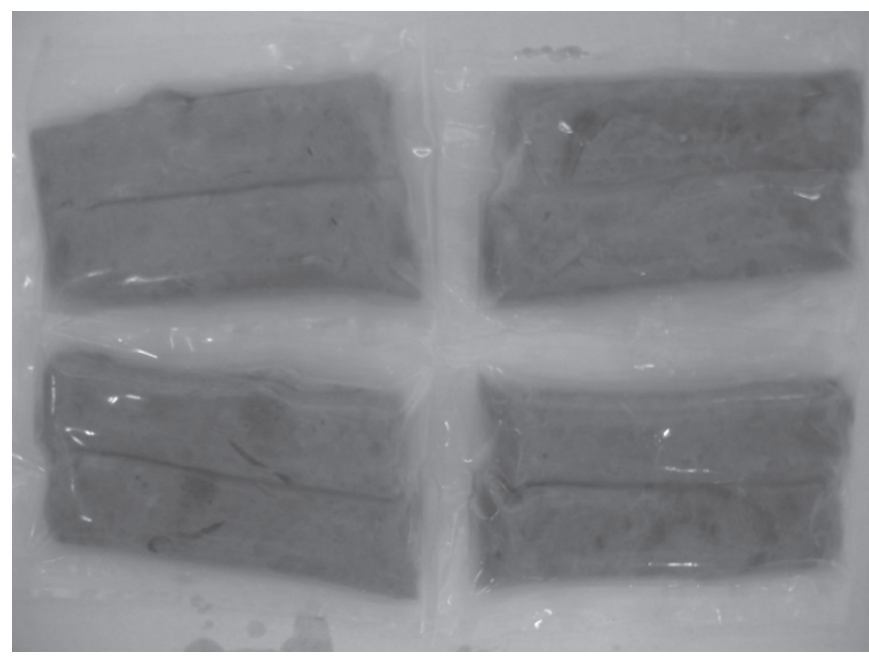

Figura 1. Presunto de peru embalado a vácuo. 
A câmara contendo o cilindro foi hermeticamente fechada e vedada para evitar vazamentos. Primeiramente, uma bomba pneumática foi acionada na câmara, injetando uma pré-carga até que seus selos se fechassem. Posteriormente, foi acionada automaticamente uma segunda bomba hidráulica que deslocou um pistão e elevou a pressão até a condição de trabalho desejado, caracterizando, assim, dois estágios de pressurização.

O equipamento operou a uma taxa de pressurização de $7 \mathrm{MPa} / \mathrm{s}$ até a pressão desejada, iniciando-se então a contagem do tempo total do processo. O tempo de processo foi contado a partir do momento em que a câmara alcançou a pressão desejada, até o início da despressurização. No decorrer do processo, pequenas oscilações de temperatura ocorreram devido à pressão ser diretamente proporcional à temperatura, tendo havido um aumento em torno de $3{ }^{\circ} \mathrm{C}$ a cada $100 \mathrm{MPa}$. Ao término do ciclo, após a despressurização, a câmara foi aberta e as amostras pressurizadas foram retiradas do cilindro e enviadas para as respectivas análises.

\subsection{Análise instrumental de cor}

A análise instrumental de cor foi realizada objetivando-se verificar possíveis mudanças, durante a estocagem, na coloração de presunto de peru controle e nas amostras submetidas às diferentes condições de pressão e tempo de pressurização. As amostras foram cortadas em pedaços com espessura de $2 \mathrm{~cm}$, modeladas em um cilindro de $2 \mathrm{~cm}$ de diâmetro, obtendose uma amostra com especificações de $2 \mathrm{~cm}$ de altura com $2 \mathrm{~cm}$ de diâmetro. Em seguida, foram embaladas a vácuo em sacos plásticos estéreis (Nasco WHIL-PACK ${ }^{\circledR}$ ), sendo posteriormente encaminhadas para o Laboratório de Análise Sensorial da Embrapa Agroindústria de Alimentos. Foi realizado um planejamento experimental $2^{2}$, com triplicata no ponto central, com pressões de 200, 300 e $400 \mathrm{MPa}$ e tempos de 5, 10 e 15 minutos à temperatura ambiente. As amostras foram analisadas durante os períodos de 1, 15, 35, 45 e 65 dias de estocagem. As coordenadas de cor foram determinadas por reflectância no $S$ \& M Colour Computer modelo SM - 4-CH da Suga, no sistema de Hunter, com abertura de $10 \mathrm{~mm}$ de diâmetro para cada amostra, e os resultados foram expressos em relação à luminosidade $\left(L^{*}\right)$ e variação da intensidade da cor vermelha $\left(\mathrm{a}^{*}\right)$. As análises foram realizadas em triplicatas com 12 leituras.

\subsection{Oxidação de lipídios}

Para as análises de oxidação de lipídios, após a pressurização na condição de $400 \mathrm{MPa}$ a 15 minutos à temperatura ambiente, baseada em estudos preliminares com presunto, em que foi avaliada a melhor condição de estocagem em relação ao crescimento de bactérias responsáveis pela deterioração. Utilizaram-se $1000 \mathrm{~g}$ das amostras controle e pressurizada, embaladas a vácuo e divididas em sacos plásticos estéreis (Nasco WHIL-PACK ${ }^{\circledR}$ ) de $20 \mathrm{~g}$ cada, que foram enviadas para o Laboratório de Análises Físico-Químicas da Universidade Federal Rural do Rio de Janeiro, no Instituto de Tecnologia. As amostras foram armazenadas a $8^{\circ} \mathrm{C}$ e analisadas a cada 15 dias, durante 90 dias de armazenamento.

\section{Índice de peróxido}

O Índice de Peróxido (IP) foi usado para medir a fase inicial da oxidação, na qual há liberação de peróxidos e as alterações não são percebidas sensorialmente. Devido à sua ação fortemente oxidante, os peróxidos orgânicos formados no início da rancificação atuam sobre o iodeto de potássio liberando iodo, que será titulado com o tiossulfato de sódio, em presença de amido como indicador. Ele indica até que ponto a oxidação progrediu. Para determinação do índice de peróxidos, utilizouse metodologia analítica do Instituto Adolfo Lutz - Métodos Físicos e Químicos para Análise de Alimentos (1985).

\section{Índice de TBA (ácido 2-tiobarbitúrico)}

O índice do ácido tiobarbitúrico (TBA) foi usado para medir a oxidação em uma fase mais avançada, na qual ocorre a produção dos compostos intermediários, reativos ao TBA e que são percebidos sensorialmente. $\mathrm{O}$ índice de TBA quantifica o malonaldeído (MDA), um dos principais produtos de decomposição dos hidroperóxidos de ácidos graxos poliinsaturados, formado durante o processo oxidativo - o MDA (CECCHI, 2003). A reação envolve o ácido 2-tiobarbitúrico com o malonaldeído, produzindo um composto de cor vermelha, medido espectrofotometricamente a $532 \mathrm{~nm}$ de comprimento de onda. A quantificação de malonaldeído é feita a partir de curvas de calibração construídas com concentrações conhecidas do reagente. Os resultados são expressos em unidade de absorbância por unidade de massa de amostra, ou em "valor de TBA" ou "número de TBA", definidos como a massa, em mg de malonaldeído por kg de amostra (ANGELO, 1996).

Para determinação do TBA, utilizou-se a metodologia analítica do Ministério da Agricultura - Métodos Analíticos para Controle dos Produtos de Origem Animal e seus Ingredientes - Instrução Normativa, $n^{\circ} 20$ de 21/07/1999. Essa metodologia fundamentase na formação de um composto de coloração vermelha resultante da condensação de 2 moles de ácido 2-tiobarbitúrico com 1 mol de aldeído malônico ou de seus tautômeros (originados na oxidação de lipídios). A quantificação de malonaldeído foi feita a partir de curvas de calibração construídas com concentrações conhecidas de malonaldeído, o padrão utilizado foi o 1,1, 3,3 - tetraetoxipropano (TEP), que, nas condições ácidas do teste, sofreu hidrólise liberando o malonaldeído.

\subsection{Análise estatística}

Para as análises dos dados de oxidação de lipídios e cor, foi realizada a técnica de análise de variância (ANOVA), seguida do teste de Tukey ao nível de 5\% de significância (VIEIRA, 2005).

Os dados de cor foram analisados por ANOVA considerando $\mathrm{p} \leq 0,05$ utilizando o software Statistica 7.0.

\section{Resultados e discussão}

\subsection{Análise instrumental de cor}

Na Tabela 2 são apresentados os valores da estatística p do teste de Dunnett referentes aos parâmetros de cor L $\mathrm{L}^{\star} \mathrm{e} \mathrm{a}^{*}$ para as amostras pressurizadas e controle, ao longo do armazenamento. 
Observou-se que o aumento da pressão e o aumento do tempo de pressurização afetaram significativamente apenas o parâmetro $\mathrm{a}^{*}$ (intensidade de vermelho), avaliado aos $45 \mathrm{e}$ 65 dias de armazenamento.

Estudos realizados por Tanzi et al. (2004) constataram que o processo de $\mathrm{APH}$ em pressões maiores que $300 \mathrm{MPa}$ provocou o decréscimo da cor vermelha de presuntos de parma, em comparação com a amostra controle. Outros estudos realizados por Tanzi et al. (2004) e Andrés et al. (2006) com presuntos cozidos e adicionados de nitrito, mostraram que devido à formação do composto estável nitroso-hemocromo a cor não foi consideravelmente afetada pela $\mathrm{APH}$ e se manteve estável durante a vida de prateleira, sugerindo que presuntos foram bons produtos para serem pressurizados. No presente estudo, entretanto, a média dos valores de $\mathrm{L}^{*} \mathrm{e} \mathrm{a}^{*}$ durante o armazenamento revelam que o aumento da pressão afetou significativamente $(\mathrm{p} \leq 0,05)$ o parâmetro $\mathrm{a}^{*}$ ao longo do armazenamento dos produtos, quando comparado com o presunto de peru controle, mostrando que o presunto de peru tratado por APH a $400 \mathrm{MPa}$ teve um decréscimo da cor vermelha (valor de $\mathrm{a}^{*}$ ), mesmo adicionado do sal de cura.

\subsection{Oxidação de lipídios}

\section{Indice de peróxido}

$\mathrm{Na}$ Tabela 3, estão representados os valores das médias e desvio padrão do índice de peróxido, analisado em triplicata para as amostras controle e pressurizada.

Analisando os resultados obtidos através do tratamento estatístico, pode-se observar que não houve diferença significativa $(p>0,05)$ entre as amostras controle e pressurizada no $30^{\circ}$ dia e houve alteração do índice de peróxido ao longo dos 90 dias a $8^{\circ} \mathrm{C}$, com diferença significativa $(\mathrm{p} \leq 0,05)$ do $15^{\circ} \mathrm{e}$ $30^{\circ}$ dia em relação aos demais.
Inicialmente ocorre a reação dos radicais livres dos ácidos graxos com oxigênio, havendo a formação dos peróxidos e hidroperóxidos, que são considerados os primeiros produtos formados na oxidação de gorduras (ARAÚJO, 1995; WONG, 1995). Segundo a legislação brasileira, o limite máximo permitido de índice de peróxido em uma amostra é de $10 \mathrm{mg}$ de peróxido $\mathrm{O}_{2} / \mathrm{kg}$. Na presente pesquisa, pode-se verificar que os valores de índice de peróxido foram abaixo deste limite máximo permitido, mas no $15^{\circ}$ e $30^{\circ}$ dia, no caso da amostra pressurizada, e no $15^{\circ}$ da amostra controle, esses valores foram superiores, indicando que houve produção do composto. Dessa forma, houve uma diferença entre as amostras no $30^{\circ}$ dia, indicando que a amostra pressurizada ainda estava produzindo peróxidos, enquanto que na amostra controle o teor detectado foi muito reduzido. Após este período, as amostras tiveram seus valores decrescidos até não haver mais a produção do peróxido, e sim de compostos intermediários, avaliados pelo índice de TBA.

\section{Indice de $T B A$}

Na Tabela 4 seguem as médias e desvio padrão do índice de TBA, analisados em triplicata para as amostras controle e pressurizada.

Tabela 3. Médias e desvio padrão do índice de peróxido, em meq de peróxido. $100 \mathrm{~g}^{-1}$, para amostras de presunto de peru controle $\mathrm{e}$ pressurizada ao longo do armazenamento.

\begin{tabular}{|c|c|c|c|c|c|c|c|c|}
\hline \multicolumn{9}{|c|}{ Tempo de armazenamento (dias) } \\
\hline Amostras & $1^{\circ}$ & $15^{\circ}$ & $30^{\circ}$ & $45^{\circ}$ & $60^{\circ}$ & $75^{\circ}$ & $90^{\circ}$ & $\begin{array}{l}\text { Desvio } \\
\text { padrão }\end{array}$ \\
\hline Controle & $0,00^{\mathrm{a}}$ & $15,04^{b}$ & $1,52^{b}$ & $0,00^{\mathrm{a}}$ & $0,00^{\mathrm{a}}$ & $0,00^{\mathrm{a}}$ & $0,00^{\mathrm{a}}$ & $\pm 5,617$ \\
\hline $\begin{array}{l}\text { Pressurizada } \\
(400-15)\end{array}$ & $0,00^{\mathrm{a}}$ & $13,25^{\mathrm{b}}$ & $12,07^{\mathrm{b}}$ & $4,88^{\mathrm{a}}$ & $0,00^{\mathrm{a}}$ & $0,00^{\mathrm{a}}$ & $0,00^{\mathrm{a}}$ & $\pm 5,982$ \\
\hline
\end{tabular}

Tabela 2. Valores de p do Teste de Dunnett para os parâmetros de cor $\mathrm{L}^{\star \S} \mathrm{e} \mathrm{a}^{\star \S \S}$ das amostras de presunto de peru ao longo da vida útil.

\begin{tabular}{|c|c|c|c|c|c|c|c|}
\hline \multicolumn{8}{|c|}{ Tratamento: Pressão (MPa) -Tempo (minuto) } \\
\hline Tempo de armazenamento & $200-05$ & $200-15$ & $300-10$ & $300-10$ & $300-10$ & $400-05$ & $400-15$ \\
\hline \multicolumn{8}{|l|}{$1^{\circ}$ dia } \\
\hline Dunnett- $\mathrm{L}^{*}$ & 0,4892 & 0,463 & 0,219 & 0,999 & 0,955 & 0,991 & 0,999 \\
\hline Dunnett $-\mathrm{a}^{*}$ & 0,980 & 0,999 & 0,987 & 0,497 & 0,730 & 0,794 & 0,441 \\
\hline \multicolumn{8}{|l|}{$15^{\circ} \mathrm{dia}$} \\
\hline Dunnett $-\mathrm{L}^{*}$ & 1,000 & 1,000 & 0,998 & 0,999 & 0,968 & 0,999 & 0,895 \\
\hline Dunnett $-\mathrm{a}^{*}$ & 0,899 & 1,000 & 0,999 & 0,958 & 0,999 & 1,000 & 0,533 \\
\hline \multicolumn{8}{|l|}{$35^{\circ} \mathrm{dia}$} \\
\hline Dunnett- $\mathrm{L}^{*}$ & 0,4333 & 0,999 & 0,999 & 0,6111 & 0,1555 & 0,410 & 0,999 \\
\hline Dunnett - $\mathrm{a}^{*}$ & 0,182 & 0,152 & 0,138 & 0,084 & 0,1292 & 0,237 & 0,152 \\
\hline \multicolumn{8}{|l|}{$45^{\circ} \mathrm{dia}$} \\
\hline Dunnett - $\mathrm{L}^{*}$ & 0,995 & 0,763 & 0,999 & 0,332 & 0,902 & 0,863 & 0,999 \\
\hline Dunnett $-\mathrm{a}^{*}$ & 0,492 & 0,831 & 0,776 & 0,999 & 0,075 & $0,0051^{\star}$ & $0,0057^{\star}$ \\
\hline \multicolumn{8}{|l|}{$65^{\circ} \mathrm{dia}$} \\
\hline Dunnett - $\mathrm{L}^{*}$ & 0,967 & 0,997 & 1,000 & 0,996 & 0,999 & 0,999 & 0,968 \\
\hline Dunnett- $\mathrm{a}^{*}$ & 0,921 & 0,988 & 0,1498 & 0,851 & 0,996 & 0,069 & $0,002^{\star}$ \\
\hline
\end{tabular}

${ }^{6} \mathrm{~L}=$ luminosidade $(0=$ preto e $100=$ branco $){ }^{\varsigma 5} \mathrm{a}=$ intensidade de verde/ vermelho $(-80 \text { até zero }=\text { verde, do zero ao }+100=\text { vermelho })^{5}$. 
Tabela 4. Médias ${ }^{\S}$ e desvio padrão de TBA, em mg de malonaldeído.kg-1 ${ }^{-1}$, para amostras de presunto de peru pressurizada e controle ao longo de 90 dias de armazenamento.

\begin{tabular}{|c|c|c|c|c|c|c|c|c|}
\hline \multicolumn{9}{|c|}{ Tempo de armazenamento (dias) } \\
\hline Amostras & $1^{\circ}$ & $15^{\circ}$ & $30^{\circ}$ & $45^{\circ}$ & $60^{\circ}$ & $75^{\circ}$ & $90^{\circ}$ & Desvio padrão \\
\hline Controle & $3,59870^{a}$ & $0,23550^{\mathrm{a}}$ & $13,15010^{\mathrm{b}}$ & $0,53120^{\mathrm{a}}$ & $1,78770^{\mathrm{a}}$ & $0,36080^{\mathrm{a}}$ & $3,08430^{\mathrm{a}}$ & $\pm 5,617$ \\
\hline $\begin{array}{l}\text { Pressurizada } \\
(400-15)\end{array}$ & $3,01670^{\mathrm{a}}$ & $0,46830^{\mathrm{a}}$ & $9,01240^{\mathrm{b}}$ & $1,38620^{\mathrm{a}}$ & $1,35980^{\mathrm{a}}$ & $0,61940^{\mathrm{a}}$ & $1,97770^{\mathrm{a}}$ & $\pm 5,982$ \\
\hline
\end{tabular}

Letras iguais em uma mesma linha e coluna, não diferem entre si significativamente ( $\mathrm{p} \leq 0,05)$, para o teste de Dunnett. ${ }^{\S}$ e o teste de média de três repetições por amostra.

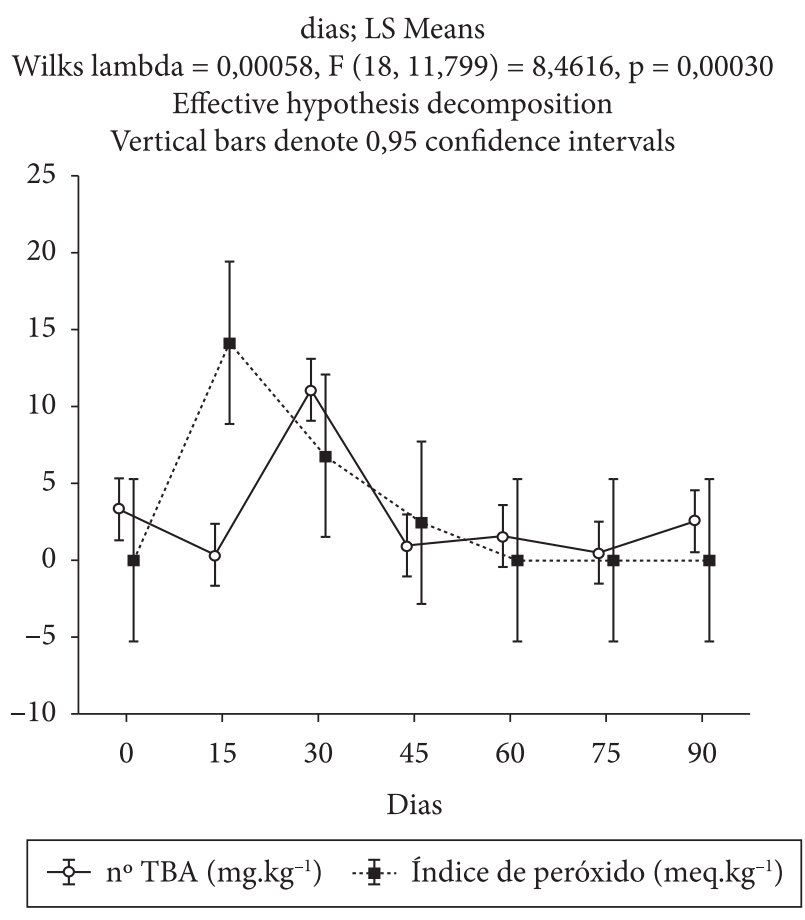

Figura 2. Médias do índice de TBA e índice de peróxido, ao longo do armazenamento.

De acordo com a Tabela 4, o valor de malonaldeído.kg-1 atingiu o ponto máximo aos 30 dias e depois apresentou um decréscimo. Analisando os resultados após análise estatística dos dados, pode-se observar que não houve diferença significativa $(\mathrm{p} \leq 0,05)$ entre as amostras controle e pressurizada.

No entanto, durante os 90 dias de armazenamento a $8{ }^{\circ} \mathrm{C}$, houve alteração do índice de TBA no $30^{\circ}$ dia em relação aos demais, com uma maior produção do ácido 2-tiobarbitúrico.

Valores de TBARS até $1,59 \mathrm{mg}$ de aldeído malônico. $\mathrm{kg}^{-1}$ de amostra são considerados baixos para serem percebidos por análise sensorial e não causam alarme para saúde do ser humano (TORRES; OKANI, 2000).

Em frangos cozidos são formados maiores teores de malonaldeído que em carnes bovinas, devido à maior composição de ácidos graxos insaturados dos primeiros. Durante a cocção ocorre fato similar, isto é, as carnes bovinas, por possuírem menores quantidades de ácidos graxos poliinsaturados, exibem menores teores de malonaldeído que as carnes de frango e suína (SIU; DRAPER, 1978).
Segundo Nunes et al. (2003), a carne de frango, tanto branca, quanto escura, apresenta sérios problemas na conservação. A oxidação lipídica, em razão da alta concentração de ácidos graxos poli-insaturados, constitui um fator preocupante, principalmente em carne escura, face ao seu alto teor em ferro e fosfolipídios.

Os lipídios são importantes componentes dos produtos cárneos, conferindo características desejáveis de suculência, sabor e aroma, valor nutricional e propriedades tecnológicas. Contudo, são facilmente oxidáveis, levando à rancificação, com a produção de toxinas indesejáveis e comprometendo a qualidade e a vida útil dos produtos. As substâncias tóxicas produzidas são cetonas, aldeídos, álcoois, ácidos e hidrocarbonetos, responsáveis pelo odor e sabor característico de ranço (OLIVO, 2006).

Comparando-se os valores de oxidação lipídica em relação ao índice de peróxido e TBA, de acordo com a Figura 2, podese observar que os peróxidos foram os primeiros compostos a serem produzidos, e, à medida que esses valores foram reduzindo, por volta do $30^{\circ}$ dia, pode-se observar que já havia a produção dos compostos intermediários, os quais tiveram sua produção máxima em torno do $30^{\circ}$ dia, que foram detectados através do índice de TBA.

\section{Conclusões}

A cor do presunto de peru avaliada instrumentalmente não se manteve estável ao longo do armazenamento. As análises mostraram que pressões de $400 \mathrm{MPa}$, após os 45 dias de armazenamento, causaram mudanças significativas na coloração do presunto de peru, especialmente em relação ao parâmetro $\mathrm{a}^{*}$ (intensidade de vermelho), tornando o produto menos vermelho. Possivelmente essa alteração tenha se dado devido à oxidação de lipídios, que alcançou seu máximo por volta do $30^{\circ}$ dia, formando compostos intermediários que levaram à alteração de cor.

Em relação à oxidação de lipídios, o produto tratado a $400 \mathrm{MPa}$ por 15 minutos à temperatura ambiente não diferiu significativamente $(\mathrm{p}>0,05)$ da amostra controle em relação ao índice de peróxido e o índice de TBA, indicando que o tratamento nessas condições não foi capaz de acelerar a oxidação de lipídios. Tais alterações seriam capazes de diminuir a vida útil do produto ocasionando a rancificação e a formação de compostos indesejáveis. 


\section{Referências bibliográficas}

ANDRÉS, A. I. et al. High pressure treatment of dry-cured Iberian ham. Effect on color and oxidative stability during chill storage packed in modified atmosphere. European Food Research and Technology, v. 222, p. 486-491, 2006.

ANGELO, A. J. Lipid oxidation in foods. Food Science Nutrition, v. 36, n. 3, p. 175-224, 1996.

ARAÚJO, J. M. A. Química de alimentos. 2. ed. Viçosa: Editora da UFV, 1999.

BORGO, L. A.; ARAÚJO, W. M. C. Mecanismos dos processos de oxidação lipídica. Higiene Alimentar, v. 19, n. 130, p. 50-57, 2005.

CECCHI, H. M. Fundamentos teóricos e práticos em análise de alimentos. 2. ed. Campinas: Editora da Unicamp, 2003.

INSTITUTO ADOLFO LUTZ. Métodos físicos e químicos para análise de alimentos. 3. ed. São Paulo: Instituto Adolfo Lutz, 1985.

NUNES, M. L. et al. Efeito de antioxidantes e das condições de estocagem na oxidação lipídica de lingüiças de frango. Revista Nacional da Carne, n. 319, p. 36-48, 2003.

OLIVO, R. Alterações oxidativas em produtos cárneos. In: SHIMOKOMAKI, M. et al. (Ed.). Atualidades em ciência e tecnologia de carnes. São Paulo: Varela, 2006. cap. 15, p. 155-162.
RUBIO, B. et al. The effects of high pressure treatment and of storage periods on the quality of vacuum-packed "salchichón" made of raw material enriched in monounsaturated and polyunsaturated fatty acids. Innovative Food Science and Emerging Technologies, v. 8, p. 180-187, 2007.

SHIMOKOMAKI, M.; OLIVO, R. Suplementação de vitamina e melhora a qualidade de carnes e derivados. In: SHIMOKOMAKI, M. et al. (Ed.). Atualidades em ciência e tecnologia de carnes. São Paulo: Varela, 2006. cap. 11, p. 115-121.

SIU, G. M.; DRAPER, H. H. A survey of the malonaldehyde content of retail meats and fish. Journal of Food Science, v. 43, p. 1147-1149, 1978.

TANZI, E. et al. High-pressure treatment of raw ham. Sanitation and impact on quality. Industria Converse, v. 79, p. 37-50, 2004.

TORRES, E. A. F. S.; OKANI, E. T. Teste de TBA: ranço em alimentos. Revista Nacional da Carne, v. 243, p. 68-76, 1997.

VIEIRA, S. Análise de variância (ANOVA). São Paulo: Atlas, 2006.

WONG, D. W. S. Química de los alimentos: mecanismos y teoría. 3. ed. Zaragoza: Acríbia, 1995. 